\title{
Il trattamento con analoghi della somatostatina è indicato in tutti i pazienti con acromegalia prima della chirurgia e, se così, per quanto tempo?
}

\author{
Salvatore Cannavò
}

Pubblicato online: 12 settembre 2014

(c) Springer International Publishing AG 2014

\section{Commento a:}

Should all patients with acromegaly receive somatostatin analogue therapy before surgery and, if so, for how long?

\section{J.J. Jacob, J.S. Bevan.}

Clin Endocrinol (2014) doi:10.1111/cen.12553

La chirurgia rappresenta il trattamento di prima linea nei pazienti con acromegalia, soprattutto se dovuta a microadenomi o macroadenomi ipofisari non invasivi. In queste condizioni le percentuali di successo sono molto elevate, soprattutto se il chirurgo può vantare una specifica esperienza. Negli altri casi, o quando un esperto neurochirurgo non è disponibile, il trattamento con analoghi della somatostatina (SSa) rappresenta una valida alternativa, permettendo il controllo dei livelli di GH e IGF-1 in un terzo dei casi e la riduzione del volume dell' adenoma ipofisario GH-secernente in quasi due terzi dei pazienti. Sebbene alcuni studi abbiano dimostrato che il trattamento con SSa prima della chirurgia migliori il profilo clinico e metabolico dei pazienti e riduca il volume dell'adenoma, le più recenti linee guida hanno concluso che non vi sono sufficienti evidenze per raccomandare un trattamento farmacologico prechirurgico.

In questo articolo Jacob e Bevan riportano le più recenti evidenze sull'argomento, valutando gli eventuali benefici anestesiologici, la riduzione degli eventi avversi chirurgici, gli effetti sulla funzione ipofisaria residua, la riduzione della degenza perioperatoria e, non ultimo, l'efficacia chirurgica, in base ai risultati di una meta-analisi, 4 studi prospettici e 6 studi retrospettivi. La revisione di tali fonti dimostra che:

S. Cannavò $(\varangle)$

Dipartimento di Medicina Clinica e Sperimentale,

Università di Messina, Messina, Italia

e-mail: cannavos@unime.it
1. sebbene alcuni studi dimostrino che il pretrattamento con SSa esercita effetti benefici sul rischio cardiovascolare, sulla sindrome delle apnee notturne e, almeno in alcuni pazienti, sul profilo glicemico, gli unici due che hanno valutato il rischio anestesiologico non hanno dimostrato significativi vantaggi;

2. in alcuni pazienti, il pretrattamento con SSa induce la riduzione del volume tumorale e rende il tessuto più soffice, facilitandone l'asportazione. Tuttavia, tre studi prospettici e randomizzati e gli studi retrospettivi non hanno dimostrato vantaggi sugli eventi avversi chirurgici. Solo uno studio prospettico ha dimostrato una riduzione della prevalenza di rinoliquorrea e un altro, poco significativo, ha evidenziato una riduzione dei tempi di degenza ospedaliera;

3. l'ipopituitarismo o il diabete insipido post-chirurgico si verificano in meno del $5 \%$ dei casi. I pochi dati disponibili al riguardo non indicano differenze significative nella insorgenza di deficit ipofisari nei pazienti pretrattati o meno con $\mathrm{SSa}$;

4. per quanto riguarda l'efficacia chirurgica, i metodi e i risultati sono ampiamente discordanti nei differenti studi. Comunque, la meta-analisi presa in considerazione dimostrerebbe che i vantaggi del pretrattamento farmacologico sono più consistenti nei centri che denunciano una minore percentuale di successo chirurgico.

In conclusione, i dati di letteratura non supportano l'indicazione di pretrattare con SSa tutti i pazienti candidati alla chirurgia. Tale approccio può essere opportuno in selezionati casi con complicanze metaboliche, cardiovascolari e respiratorie, per ridurre il rischio perioperatorio. Il pretrattamento con SSa può essere indicato anche nei pazienti con macroadenoma ipofisario, soprattutto se invasivo e nei centri con percentuali di successo chirurgico $<50 \%$. Il periodo di pretrattamento con SSa dovrebbe essere di 3-6 mesi. 